\title{
Unit Maintenance Scheduling Coordination Mechanism in Electricity Market Environment
}

\author{
Gang $\mathrm{Lu}^{1,2}$, C.Y. Chung ${ }^{2}$, K. P. Wong ${ }^{2,4}$, Fushuan Wen ${ }^{3}$ \\ 1: Department of Electrical Engineering, Zhejiang University, Hangzhou, China. (lugang.ee@gmail.com) \\ 2: Department of Electrical Engineering, The Hong Kong Polytechnic University, Hong Kong. \\ 3: College of Electrical Power, South China University of Technology, Guangzhou, China. \\ 4: On leave with School of Electrical, Electronic and Computer Engineering, University of Western Australia, \\ Australia
}

Abstract-- Along with worldwide electricity market deregulation, the traditional approach to unit maintenance scheduling (UMS) is required to make compatible changes for resolving some new challenges. The development of workable coordination mechanism to accord with the market mechanism is under the close concern of the policymaker, the market designer and the market participants. Actually, the key of this problem is both how to treat generation companies equally and how to guarantee the system security during the UMS process. This paper advances the view on UMS in electricity market environment, and identifies that the coordination fairness should include "which plans to be selected for adjustment" and "how to adjust them" simultaneously, of which only the former was comprehensively studied in the past research, however. To ensure the competition fairness thoroughly, a new kind of UMS mechanism with a coordination model is constructed in this paper. The transmission capacity constraint and random line outage are involved in the model in order to make the analysis about the impact of UMS on the system security more reasonable. In addition, the adequate solution for this model and the allocation way about the ISO's income which is created during UMS process, have also been discussed. Then the proposed mechanism is applied to a numerical example to show its good features in practical application.

Index Terms--congestion rate; cost allocation; coordination coefficient; electricity market; fairness; unit maintenance scheduling

\section{INTRODUCTION}


In the traditional vertical bundled power systems, unit maintenance scheduling (UMS) is centrally dispatched based on the system security and economy [1-4]. However, in the new competitive market environment, the unit maintenance cost depends on not only the maintenance expense, but also the electricity price of the market. Generation companies (GENCOs) prefer the maintenance time with lower price in order to reduce their financial losses of selling energy. Some techniques [5-7] have been recently proposed for GENCOs to determine their own optimal UMS in the market environment. Although it sounds reasonable for GENCOs to decide UMS on the grounds of market mechanism, the system security may be deteriorated if the UMS is not well coordinated, - the electricity shortage in the California market in winter 2000 partly caused by the individual arrangement of UMS as an example. As a result, the independent system operator (ISO) must coordinate the maintenance scheduling of GENCOs to guarantee the system security. In fact, there still exist disputes about whether UMS should be determined centrally or by market-based methods, i.e. the competition and coordination [8]. The sensible choice should consider both the generation capacity adequacy and the market development stage. For instance, the market-based method is adopted in the Spanish [9], Nordic and British [10] market while the central arrangement is accepted in China currently, but the market-based method is more acceptable for the market participants in the long run. Arrangement of UMS in the market environment has attracted extensive attention. The Federal Energy Regulatory Commission (FERC) of USA has identified this problem in its Wholesale Power Market Platform White Paper issued on April 28, 2003. It is important that the balance can be kept between affording competition opportunity and essential coordinating in the deregulation for maintenance arrangement.

As mentioned above, coordination about UMS, which is an important matter both for GENCOs' interest and the system security, must ensure fairness and reasonableness, i.e. treating GENCOs equally and guaranteeing the system security. Some coordination mechanisms were suggested in [9, 11-15], which can be classified as the following three categories:

1) The ISO coordinated UMS based on both GENCOs' interest and the system security. In [11], the coordination object was both the minimal fluctuation of the reserve rate at all the windows and the minimal fluctuation of the window variance for maintenance of all the units. In [12], it considered both the minimal Expected Electricity Not Supplied (EENS) and the maximal total GENCOs' satisfaction degree measured by fuzziness. However, the way in $[11,12]$ failed to ensure fairness and could not settle arguments among GENCOs.

2) The ISO negotiated UMS with GENCOs on behalf of customers and obtained the improved system security through paying for GENCOs who would adjust their plans, and the cost burden would go to customers. In [9], the ISO compared its own plan for maximizing the system reliability with the submitted ones by GENCOs, and afterwards collected positive/negative expense from GENCOs in order to prevent/encourage them to arrange 
unit maintenance at those windows with great difference. Then GENCOs submitted their plans again under the positive/negative expense. This process was repeated until the difference was fine enough. The system reliability was improved finally and the expense should be afforded by customers. This method conformed to the market-based mechanism. Unfortunately, this method suffered some applicable problems, that the coordination process was very complicated, and that it was possible for some GENCOs to exercise the market power to force the ISO to raise the positive expense.

3) The ISO coordinated UMS according to some forms of expression about maintenance desire announced by GENCOs. In [13], the ISO selected plans for adjustment by the order of Willingness-to-Pay (WTP), but adjusted them to the nearest permitted windows, hence it could not ensure fairness about "how to adjust plans" although it could ensure fairness about "which plans to be selected for adjustment". In [14], GENCOs bade for all the maintenance windows, and the ISO determined the arrangement according to collecting maximal bid from GENCOs. In fact, GENCOs participated in competition for all the windows simultaneously, but only needed to pay for the arranged windows. Hence this way will be more favorable for the units with larger capacity because of their bigger bidding at many windows. Thus this will cause unfairness and objection in application. And the WTP way and the bidding way for voicing GENCOs' will are the same in essence, only with difference in appellation.

The third category method is closer to reality, and moreover, is more convenient, flexible and applicable. But how to ensure coordination fairness and how to allocate the ISO's income still remained unsolved. Coordination fairness includes both "which plans to be selected for adjustment" and "how to adjust them", on which Reference [15] made some preliminary research. The disputes about the income allocation mainly lay in the allocation object, i.e. whether GENCOs who participate in competition for UMS in $[13,15]$, or the GENCOs whose units are unable to be scheduled for maintenance in [14], should be the one. In fact, the reason why GENCOs have additional loss in the UMS is that they need meet the load in essence, and hence the loss should be born by customers based on the theory that anyone who creates the cost should bear it, as the allocation way in [9]. On the contrary, the ISO's income during UMS is a kind of in-fighting cost for the purpose of fairness competition, so the allocation object should be all the GENCOs that participate in the competition, no matter whether their units are arranged, but the allocation method is valuable in [14].

The transmission capacity constraint has not been considered in these literatures when the impact of UMS on system security is analyzed. Actually when transmission congestion happens, electricity may not be delivered to customers even if the generation capacity is enough. Such condition generally happens in the weak transmission links. In addition, the random line outage is also a negative factor for system security. Hence the transmission should be taken into 
consideration in the coordination [16].

For solving the above problems, this paper constructs a new unit maintenance scheduling coordination model in electricity market environment, which ensures fairness that includes both "which plans to be selected for adjustment" and "how to adjust them" through an enough big coordination coefficient. Moreover, when analyzing the impact of UMS on system security, we do not only take the generation capacity adequacy, but also the transmission capacity constraint including the random line outage into account through adopting the ' $\mathrm{N}-1$ ' examination of lines and the concept of congestion rate (CR). In addition, the adequate solution for this model and the allocation method will be discussed. Finally, a numerical example is presented to illustrate the essential features of the proposed method.

\section{UNIT MAINTENANCE SCHEDULING IN MARKET ENVIRONMENT}

\section{A. Arrangement Process of UMS and Coordination Model}

In this study, the interval is assumed to be one year consisting of 52 weekly maintenance windows, but the proposed method can be used for longer or shorter windows.

A good coordination mechanism should be accepted by GENCOs and permitted by system security. Hence on the one hand, the ISO should be neutral, which means it should only be required to determine condition on which the system is reliable and need not seek to improve the system security when coordinating the UMS after that. Obviously, it may set much stricter constraints if called for more reliable system. And on the other hand, only when GENCOs may voice their will during participating in both competition processes, i.e. "which plans to be selected for adjustment" and "how to adjust them", can the competition fairness among GENCOs be ensured by the ISO as an independent neural participant in theory. As a result, the arrangement process this paper suggests is as follows:

\section{1) GENCOs Submit Their Own Plans:}

GENCOs are required to submit their own unit maintenance plans including the maintenance capacity, the maintenance duration, the available windows for maintenance and the maintenance Willingness-to-Pay curve in the agreed time, as shown in Fig. 1. WTP is adopted in this paper as the form of voicing the strength of GENCOs' will about the maintenance windows. The more WTP is, the stronger is the will. And hence WTP curve may show a 
GENCO's different will about different plans. The different preference is determined in terms of their own unit characteristic, e.g. the maintenance record, the maintenance expense, the operation plans and the forecasting electricity price in the future. Obviously, the best will for maintenance windows voiced by this GENCO, who requires three weeks for maintenance duration, is the $19^{\text {th }} \sim 21^{\text {st }}$ week with maximal WTP in Fig. 1 , and the $20^{\text {th }} \sim 22^{\text {nd }}$ week is its second best choice with the second maximal WTP, and the rest ranking can be deduced in this way. So the WTP curves can reveal GENCOs' will apparently. Certainly, these submitted plans need to be justified by the ISO, and the illegal ones, e.g. beyond the available windows, will be denied. Additionally, each GENCO may consider others' strategies when determining its own submitted UMS, and hence the game among the GENCOs cannot be avoidable and will be played through their submitted WTP for maximizing respective profit. However, GENCOs may be more prudent due to the additional cost of WTP.

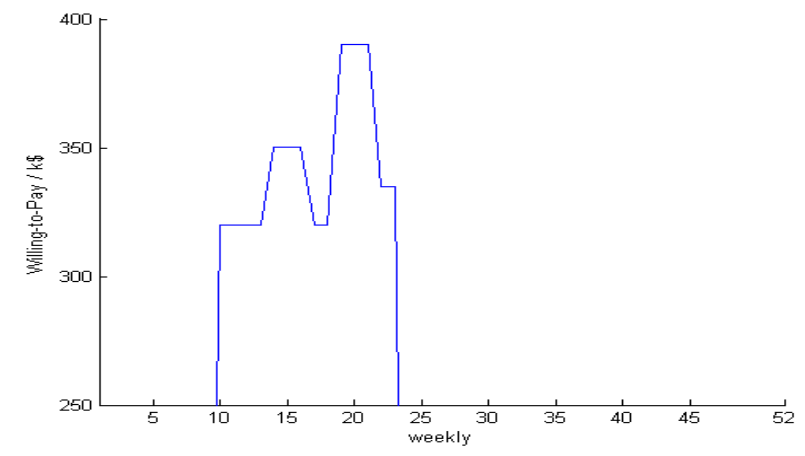

Fig. 1. Unit Maintenance Willingness-to-Pay Curve

\section{2) The ISO Coordinates Plans:}

The ISO measures the GENCOs' satisfaction degree (SD) at each possible window for maintenance, by adopting the following formula:

$$
u_{i}(x)=R\left\{\sum_{k=0}^{T_{i}-1} p_{i}\left(x_{i}+k\right) \mid x_{i}=1,2, \cdots, 52-T_{i}+1\right\}
$$

where $i$ is the unit number; $x$ is the starting window for maintenance; $u$ is the measured $\mathrm{SD}$; $T$ is the maintenance duration; $p$ is the WTP $(\$)$ per window; $R$ is the descendent rank operation about the elements of the set $\{\cdot\}$, and the 
results corresponding with the rank position of elements are $52,51,50, \ldots$, and the measured value is equal if WTP is the same. From (1), each GENCO's SD ranking about different plans is measured fully according to its submitted WTP curves, and some GENCOs will have the same SD even though they submitted different WTP for one common maintenance window, accordingly this measured way about the SD will not affect the fairness of the following coordination process.

The ISO arranges the UMS according to the following coordination model:

$$
\begin{gathered}
\max \sum_{i=1}^{N} \sum_{k=0}^{T_{i}-1} p_{i}\left(x_{i}+k\right)+W \sum_{i=1}^{N} u_{i}\left(x_{i}\right) \\
\text { s.t. } \quad \underline{x}_{i} \leq x_{i} \leq \bar{x}_{i}-T_{i} \\
A_{t}\left(\left\{x_{i}+l_{i}=t\right\}\right) \geq 0 \\
\gamma_{t}\left(\left\{x_{i}+l_{i}=t\right\}\right) \leq \gamma_{0} \\
l_{i}=0,1, \cdots, T_{i}-1 \\
x_{i} \in\{1,2,3, \cdots, 52\} \\
t=1,2,3, \cdots, 52
\end{gathered}
$$

where $N$ is the number of units; $W$ is the coordination coefficient; $\underline{x}$ and $\bar{x}$ are the lower and upper limitation of the available windows for maintenance respectively; UMS should satisfy the system generation capacity adequacy requirement of (4) and the transmission limitation conditions of (5), which will be explained in detail in Section III.

The target function of the coordination model is explained as follows. Firstly, the former part of (2) represents that the ISO puts the submitted plans into consideration one by one according to the descension of WTP for testing the power system reliability when their SD are the same. If the reliability criteria cannot be met, the last reviewed plan will be denied, and then we continue to consider the residuary in sequence until all the submission are examined. Secondly, with $W$ so big that the latter part of (2) is much bigger than the former, meeting the will of GENCOs is ensured to be the prior target. In other words, it is similar to the scenario that GENCOs submit plans by rounds and need do it again 
when their plans are denied, but the new plans cannot affect those accepted ones. Hence the big enough $W$ can avoid the situation that large capacity units may take advantage of the maintenance competition, and this method can ensure coordination fairness, which is easy to be accepted. Thirdly, the coordination coefficient shows the interest negotiation among GENCOs, which may be determined by all the GENCOs, but the bigger one can ensure fairness.

\section{3) Allocation of the ISO's Income:}

The ISO will collect WTP from GENCOs corresponding to the windows in which their submitted plans are accepted, and will allocate the whole income among GENCOs due to its own nonprofit characteristic after UMS will have already been performed in the future.

From the above arrangement process, the following conclusions can be drawn. Firstly, the ISO can guarantee the system security through determining the parameters in (4) and (5), which will be published for the market participants. Secondly, WTP submitted by GENCOs may make ISO fairly select plans for adjustment. Thirdly, the measured SD and the big enough coordination coefficient make the ISO fairly adjust those selected plans. As a matter of fact, the ISO is only responsible for the system security, and need not worry about the disputes about equality among GENCOs because the competition for maintenance is fully determined by the submitted plans for this method, and then the goal of fairness and reasonableness can be achieved naturally.

\section{B. Solution for the Coordination Model}

Many optimal techniques, such as Genetic Algorithm, may solve this coordination optimization model, but the solution process may be streamlined, based on the above explanation about the big enough coordination coefficient $W$. There are mainly two simplification ways.

On the one hand, the ISO begins to examine GENCOs' plans with the common maximal SD which is equal to 52 simultaneously for maintenance arrangement. And if some plans are denied due to the system security, they will be taken into consideration by the next smaller SD, but cannot affect those accepted ones with the larger SD. Hence the 
coordination model may be optimized in descending order of the SD, in other words, the SD determines the search direction for optimization.

On the other hand, under some common SD, some plans have overlaps in maintenance windows. Hence those plans that have no overlap in one another may be arranged firstly. And others are divided into sub-set according to the different overlap windows and will be centrally optimized respectively. As a result, the optimization dimension may be reduced.

But in reality the obtained solution by the above way may have no ability to arrange all the units, so some treatments are made. Firstly, the above obtained solution is adjusted as the initial outcome. Secondly, those un-scheduled units are arranged in the windows when they are denied for the last time only because of their less WTP. Thirdly, the coordination model of $(2) \sim(8)$ for other units is optimized again.

\section{ANALYZING THE IMPACT OF UMS ON SYSTEM SECURITY}

There are many requirements for system reliability but not all the criteria are necessary to be considered for the power system mid-term planning. Here the generation capacity adequacy and the transmission capacity constraint are considered only in this paper when analyzing whether the system is reliable with the given maintenance plans.

\section{1) Generation Capacity Adequacy:}

The surplus generation capacity index (SGCI) [13] is applied to testing the supply adequacy. Because of being measured by MW, it is selected for analysis convenience instead of other indexes, such as LOLP (lost of load probability). The SGCI is defined as follows:

$$
A=C s-C m-D-r_{0} D
$$

where $A$ is the SGCI (MW) in the maintenance window; $C s$ is the total generation capacity of the system (MW); $C m$ is the maintenance capacity (MW); $D$ is the peak load (MW); and $r_{0}$ is the lowest capacity margin (\%) which is $7 \% \sim 10 \%$ 
generally. If $A$ is smaller than zero, it means the plans may cause the risk of short supply in the maintenance window.

\section{2) Transmission Capacity Constraint:}

Unit maintenance will change the system's power flow, and may cause the overload in some weak links in the transmission. In fact, the ISO may collect data including the weekly peak loads, the network configuration, the line parameters and the capacity and distribution of units. Even though the bus load can be forecasted by the historical data, the power flow will still change with different unit commitment and the random line outage, and hence whether the transmission capacity constraint is violated still remains unknown.

Certainly, the optimal power flow (OPF) model may be applied to analyzing the impact, i.e. searching the optimal solution for realizing some optimization targets, such as the cheapest unit commitment under the transmission constraint. If there are solutions, it means the reasonable unit commitment exists. And if no solution exits, it means there is no any reasonable one and the UMS should be denied. But this method only explains whether some reasonable unit commitment exists, but cannot give other information so as that the ISO cannot know the system condition. In other words, OPF has a solution and UMS will be accepted even if only one reasonable operation condition exists for the system, but actually the ISO has inadequate dispatching ways and the system is too weak, hence the OPF model is inapplicable for analyzing the maintenance plans.

In the actual environment, the unit commitment is dispatched by the ISO according to the system security and economy. The ISO may select one from those reasonable dispatching ways in the future. Obviously, the more ways the ISO may select, the better the system security will be. It is surely the best if the system is reliable in any one. However, it is unnecessary for the actual situation. In fact, all the ISO needs are just enough acceptable ones for selection in the future. This paper adopts the concept of congestion rate (CR) [15] to evaluate the probability of unacceptable dispatching ways for the ISO in the future. CR is represented by $\gamma$ as follows:

$$
\gamma=\operatorname{Pr}\left\{f>f_{\text {max }}\right\}
$$


where $\operatorname{Pr}\{\}$ represents the probability calculation; $f$ is the line power flow; and $f_{\max }$ is the line power flow limitation.

The result shows the possibility of invalidation for all the dispatching ways. The bigger $\gamma$ is, the worse the situation is.

Then if $\gamma$ is bigger than some critical value, e.g. $\gamma_{0}$, it is recognized that the number of reasonable dispatching ways is too short for the ISO. $\gamma_{0}$ is a kind of system security index, whose value is required to be researched in practice, and here $\gamma_{0}$ is assumed as $70 \% \sim 80 \%$. The relevant plans will be denied if the CR cannot fulfill the formula, $\gamma \leq \gamma_{0}$.

In addition, when the impact of UMS on system security is investigated, the random line outage should also be taken into consideration, for which the $\mathrm{N}-1$ examination is the most usual way. UMS belongs to the mid-term planning, and hence the statistic security analysis is applicable to the N-1 examination of lines. For the large and complicated power system, it is appropriate to identify the network as some sub-systems based on the characteristic of network configuration and the distribution of units and load. Then it is only required to analyze the main joint lines among the sub-systems. The transformation about (10) is made:

$$
\gamma_{N-1}(l)=\operatorname{Pr}\left\{f>f_{\max }\right\}
$$

where $\gamma_{N-1}(l)$ represents the $\mathrm{CR}$ when the line $l$ is outage. CR can be calculated by the integrated approach of Monte Carlo method and DC power flow method in the Appendix. For the accepted UMS under N-1 examination, the maximum value of $\gamma_{N-1}(l)$ must be less than or equal to $\gamma_{0}$, i.e. $\max \left\{\gamma_{N-1}(l) \mid l \in L\right\} \leq \gamma_{0}$, where $L$ represents the set of key lines determined according to the practical condition of the whole system.

\section{ALLOCATION ABOUT THE ISO'S INCOME}

The ISO will collect WTP from GENCOs during the UMS and needs to allocate the whole income for its own nonprofit characteristic. In [9], the cost is considered to be born by customers because the ISO pays GENCOs for improving the system security. But in this paper WTP is a kind of in-fighting cost for fairness competition, hence the allocation object should be GENCOs that participate in competition.

During the UMS, GENCOs face two kinds of loss, i.e. the loss due to adjustment for their plans and the loss about 
WTP. In fact, WTP is a kind of voluntary cost for GENCO to purchase the maintenance windows, while the other is a kind of "passive" cost for satisfying system security requirement. And moreover, the adjustment process is determined actually by the submitted WTP, in other words, some GENCOs give high WTP in exchange of adjustment for others' plans which leads to the relevant loss. Accordingly, the collected WTP should give compensation for the passive loss firstly. And then if there is still surplus, the residual should compensate for GENCOs' loss about WTP. We denote $R$ as the compensation for the part of loss on account of adjustment for GENCOs' plans against their best will, and $R^{\prime}$ as the compensation for the other loss arising from competition. Based on the proportion allocation method, the income may be allocated according to (12) firstly and then the surplus should be allocated according to (13) if there is any:

$$
\begin{gathered}
R_{i}=\min \left(\sum_{i=1}^{N} \sum_{k=0}^{T_{i}-1} p_{i}\left(x_{i}^{*}+k\right), \sum_{i=1}^{N} \max \left(s_{i}, 0\right)\right) \times \frac{\max \left(s_{i}, 0\right)}{\sum_{i=1}^{N} \max \left(s_{i}, 0\right)} \\
R_{i}^{\prime}=\max \left(\sum_{i=1}^{N} \sum_{k=0}^{T_{i}-1} p_{i}\left(x_{i}^{*}+k\right)-\sum_{i=1}^{N} \max \left(s_{i}, 0\right), 0\right) \times \frac{\sum_{k=0}^{T_{i}-1} p_{i}\left(x_{i}^{*}+k\right)}{\sum_{i=1}^{N} \sum_{k=0}^{T_{i}-1} p_{i}\left(x_{i}^{*}+k\right)}
\end{gathered}
$$

where $x^{*}$ is the arranged starting window for maintenance; and $s$ is the loss due to adjustment for plans against their best will and only GENCOs with the positive value of $s$ are compensated as (12).

\section{NUMERICAL EXAMPLE AND ANALYSIS}

The modified IEEE 30-bus system, as shown in Fig. 2, is used to explain the UMS method in this paper. The system consists of eight units that are required to be scheduled for maintenance within one year. The unit number, the bus number, the maintenance capacity, the maintenance duration and the available windows for maintenance are described in Table I, and the submitted WTP curves are shown in Fig. 3. The total installed capacity is 1900 MW and the supply capacity outside the system is $750 \mathrm{MW}$ at most through bus 6 . The weekly peak loads and the LDC are the same with [15]. It is assumed that every line has a power flow limitation of $610 \mathrm{MW}$, and that $r_{0}$ and $\gamma_{0}$ are $7 \%$ and $70 \%$ respectively. And the set $L$ of lines for N-1 examination is $\{4-6,4-12,6-10,6-28,12-15,27-28\}$ according to the 
analysis on the distribution of generation capacity and peak load.

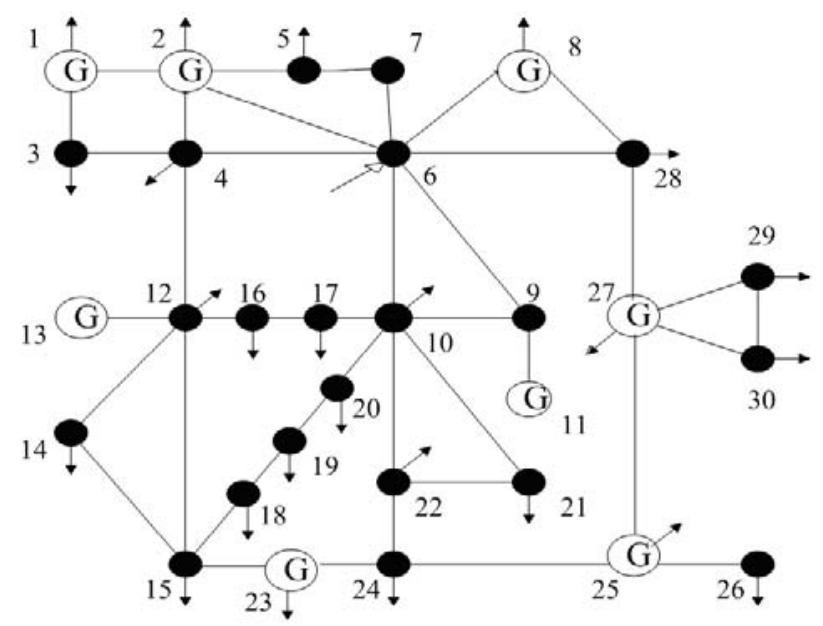

Fig. 2. Modified IEEE 30-Bus System

Based on the above explanation about the suitable solution for the coordination model, the set of units $5,6,7$ and units $1,2,4,8$ may be coordinated respectively according to (2) (8) because there exist overlaps in the maintenance windows for each set. Here, $W$ is set as a very large value, e.g. $10^{6}$, in other words, the latter part of (2) is much bigger than the former and hence maximizing GENCOs' SD is the preponderant target. The optimal UMS is shown in Table II.

The computation time of the test case is 136 seconds.

Table I

UNIT MAINTENANCE PARAMETERS

\begin{tabular}{ccccccccc}
\hline Unit no. & 1 & 2 & 3 & 4 & 5 & 6 & 7 & 8 \\
\hline Bus no. & 1 & 2 & 27 & 8 & 11 & 13 & 23 & 25 \\
\hline $\begin{array}{c}\text { Maintenance capacity } \\
(\mathrm{MW})\end{array}$ & 100 & 350 & 300 & 400 & 150 & 300 & 100 & 200 \\
\hline $\begin{array}{c}\text { Maintenance duration } \\
\text { (Week) }\end{array}$ & 2 & 4 & 4 & 4 & 2 & 4 & 2 & 3 \\
\hline $\begin{array}{c}\text { Available Windows } \\
\text { (Week) }\end{array}$ & $31^{\text {st }} \sim 46^{\text {th }}$ & $29^{\text {th }} \sim 40^{\text {th }}$ & $19^{\text {th }} \sim 32^{\text {td }}$ & $33^{\text {rd }} \sim 43^{\text {rd }}$ & $5^{\text {th }} \sim 20^{\text {th }}$ & $6^{\text {th }} \sim 17^{\text {th }}$ & $6^{\text {th }} \sim 18^{\text {th }}$ & $33^{\text {rd }} \sim 44^{\text {th }}$ \\
\hline
\end{tabular}

In this numerical example, $r_{0}$ and $\gamma_{0}$ are $7 \%$ and $70 \%$ set by the ISO respectively. Certainly, the ISO may obtain better security margin for the power system requirement through the strict selection of the two parameters. So in the 
electricity market environment, the coordination mechanism can ensure reasonableness with the ability of guaranteeing the system security, which may be accepted by the market designer and the policymaker.

Fig. 3 shows that the most favorable maintenance window for unit 1 is the $40^{\text {th }} \sim 41^{\text {st }}$ week when it has the same SD with units 4 and 8 respectively, but the simulation results identify their values of $A$ in (9) to be $-77.7 \mathrm{MW}$ in the $40^{\text {th }}$ week and $-43.6 \mathrm{MW}$ in the $41^{\text {st }}$ week respectively and moreover the total WTP of unit 1 is $\$ 100 \mathrm{k}$ for the $40^{\text {th }} \sim 41^{\text {st }}$ week, which is smaller than that of other units, so the ISO selects the plan of unit 1 for adjustment. Similarly, the common favorable maintenance window of units 5,6 and 7 is the $13^{\text {th }}$ week, but they cannot be satisfied simultaneously because $\gamma_{N-1}$ is $100 \%$ if all of them are arranged to be maintained in this week. Although the total WTP of unit 6 is $\$ 300 \mathrm{k}$ for the $10^{\text {th }} \sim 13^{\text {th }}$ week, bigger than others', the ISO accepts units 5 and 7 for maintenance instead of unit 6 . It is because $\gamma_{N-1}$ is $11.81 \%$ when units 5 and 7 are in maintenance simultaneously while $\gamma_{N-1}$ is $96.56 \%>70 \%$ even if only unit 6 is in maintenance. It is obvious that "which plans to be selected for adjustment" is determined by WTP and system security that has a close relationship with both $A$ and $\gamma$, hence the selection fairness is ensured naturally.

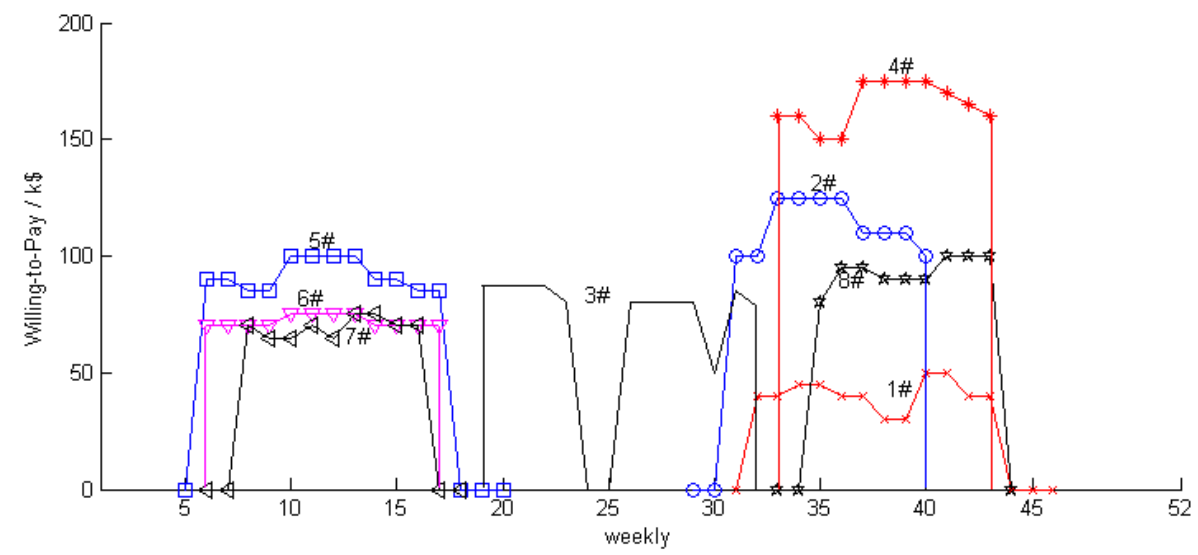

Fig. 3. Submitted Willingness-to-Pay Curves

Units 1 and 6 have to adjust their maintenance plans, as means they cannot be arranged in their most favorite windows with the SD valuing 52. So their hypo-preferred windows need to be considered for maintenance in the descending order. Unit 1 will be taken as an example to detail the analysis on the adjustment process. Both the $34^{\text {th }}$ 
$\sim 35^{\text {th }}$ and $41^{\text {st }} \sim 42^{\text {nd }}$ week are the second best option for unit 1 with the SD valuing 51 . Unfortunately, the latter is obviously an unreasonable one according to the above simulation result of $A$ in the $41^{\text {st }}$ week. Similarly, unit 1 cannot carry out maintenance in the former either, because it conflicts with unit 2 holding higher WTP in the $34^{\text {th }}$ week when $A$ is $-90.87 \mathrm{MW}$. So we consider the third best windows for unit 1 with the SD valuing 50 , i.e. $35^{\text {th }} \sim 36^{\text {th }}$ week, and unit 1 may be arranged in this term because the system security can be guaranteed. In the similar way, the plan of unit 6 can also be adjusted and arranged in the $7^{\text {th }} \sim 10^{\text {th }}$ week fairly. Actually, the WTP of unit 6 is bigger in the majority windows, but the SD corresponding to the arranged windows is only 48 even lower than other units' with smaller WTP. From the above adjustment process, it is obvious that "how to adjust plans" is determined by the will sequence of GENCOs about maintenance, and hence this method can meet GENCOs' will to the greatest extent, in addition, it may avoid the possibility of favoring units with larger capacity, so the adjustment fairness is also ensured naturally. In fact, the enough big coefficient $W$ in the coordination model makes all the GENCOs compete for maintenance in rounds, like the way of [15], so as to ensure fairness in nature.

Table II

UNIT MAINTENANCE SCHEDULING AND THE RELEVANT SYSTEM STATE

\begin{tabular}{c|c|c|c|c|c}
\hline Unit no. & $\begin{array}{c}\text { Maintenance } \\
\text { windows } \\
(\text { week })\end{array}$ & $\begin{array}{c}\text { WTP } \\
(\mathrm{k} \$)\end{array}$ & SD & $\begin{array}{c}\text { Minimal } A \\
(\mathrm{MW})\end{array}$ & $\begin{array}{c}\text { Maximal } \gamma_{N-1} \\
(\%)\end{array}$ \\
\hline 1 & $35^{\text {th }} \sim 36^{\text {th }}$ & 85 & 50 & 38.6 & 0 \\
2 & $33^{\text {rd }} \sim 36^{\text {th }}$ & 500 & 52 & 38.6 & 0 \\
3 & $19^{\text {th }} \sim 22^{\text {nd }}$ & 350 & 52 & 186.5 & $29.58 \%$ \\
4 & $37^{\text {th }} \sim 40^{\text {th }}$ & 700 & 52 & 14.8 & 0 \\
5 & $12^{\text {th }} \sim 13^{\text {th }}$ & 200 & 52 & 330.6 & $11.81 \%$ \\
6 & $7^{\text {th }} \sim 10^{\text {th }}$ & 285 & 49 & 375.9 & $68.19 \%$ \\
7 & $13^{\text {th }} \sim 14^{\text {th }}$ & 150 & 52 & 330.6 & $11.81 \%$ \\
8 & $41^{\text {st }} \sim 43^{\text {rd }}$ & 300 & 52 & 42.5 & $61.84 \%$ \\
\hline
\end{tabular}

During the maintenance arrangement, the ISO's income is $\$ 2570 \mathrm{k}$ totally, which should be allocated among GENCOs according to (12) and (13) after all the units will have finished maintenance. Being lack of practical data, we assume loss $s$ of units 1 and 6 listed in Table III. And the relevant compensation values of $R$ in (12) for the two units are 
also shown in Table III. Then there is still surplus of $\$ 1870 \mathrm{k}$, which will be allocated according to the loss proportion of WTP, as the results $R^{\prime}$ in Table III. The allocation outcome shows that each GENCO can obtain a certain sum of compensation and the losses of GENCOs whose plans are adjusted can be reduced at most. Hence, the allocation way is feasible.

Table III

LOSS AND COMPENSATION

\begin{tabular}{c|c|c|c|c|c}
\hline Unit no. & $\begin{array}{c}\text { Loss } s \\
(\mathrm{k} \$)\end{array}$ & $\begin{array}{c}\text { Loss WTP } \\
(\mathrm{k} \$)\end{array}$ & $\begin{array}{c}\text { Compensation } R \\
(\mathrm{k} \$)\end{array}$ & $\begin{array}{c}\text { Compensation } R^{\prime} \\
(\mathrm{k} \$)\end{array}$ & $\begin{array}{c}\text { Total compensation } \\
(\mathrm{k} \$)\end{array}$ \\
\hline 1 & 200 & 85 & 200 & 61.85 & 261.85 \\
2 & 0 & 500 & 0 & 363.81 & 363.81 \\
3 & 0 & 350 & 0 & 254.67 & 254.67 \\
4 & 0 & 700 & 0 & 509.34 & 509.34 \\
5 & 0 & 200 & 0 & 145.53 & 145.53 \\
6 & 500 & 285 & 500 & 207.37 & 707.37 \\
7 & 0 & 150 & 0 & 109.14 & 109.14 \\
8 & 0 & 300 & 0 & 218.29 & 218.29 \\
\hline
\end{tabular}

\section{CONCLUSIONS}

The proposed UMS coordination mechanism in this paper can realize the balance between affording competition opportunity and essential coordinating, which may provide important reference for the policymaker or the market designer. It permits GENCOs to express their will of maintenance, and can guarantee system security through the reasonable adjustment for submitted plans in the electricity market. Besides, this coordination mechanism can meet GENCOs' will to the greatest extent under treating them equally during the two identified processes, "which plans to be selected for adjustment" and "how to adjust them", so it can avoid GENCOs' arguments about coordination fairness. And moreover, considering both the generation capacity adequacy and the transmission capacity constraint including the random line outage advances the analysis about the impact of UMS on the power system. In addition, the suggested allocation way identifies the priority of compensation about two losses so as to show more sound and feasible. In summary, this method can ensure fairness and reasonableness. Our theoretical and numerical illustration has 
demonstrated that the proposed UMS method has good practicability including favorable characteristics, such as the simple theory, fast calculation and convenience implementation. But some problems still remain for future research, such as the analysis of GENCOs' game about maintenance plans, the mid-term generation plan's impact on the UMS, and how to extend the proposed method to the maintenance scheduling including both the units and the transmission lines.

\section{ACKNOWLEDGMENTS}

This work was supported by The Hong Kong Polytechnic University under the joint-supervision scheme of PhD students with Zhejiang University (Project number: G-U096).

\section{REFERENCES}

[1] Marwali, M. K. C., and Shahidehpour, S. M.: 'Long-term transmission and generation maintenance scheduling with network, fuel and emission constraints', IEEE Transactions on Power Systems, 1999, 14, (3), pp. 1160-1165

[2] Leou Rongceng: 'A flexible unit maintenance scheduling considering uncertainties', IEEE Transactions on Power Systems, 2001, 16, (3), pp. 552-559

[3] Anders, G., Hamoud, G., da Silva, A. M. L., and da Fonseca Manso, L. A.: 'Optimal outage scheduling-example of application to a large power system', International Journal of Electrical Power \& Energy Systems, 2005, 25, (8), pp. $607-614$

[4] Leou Rongceng: 'A new method for unit maintenance scheduling considering reliability and operation expense', International Journal of Electrical Power \& Energy Systems, 2006, 28, (7), pp.471-481

[5] Kim, J. H., and Park, J. K.: 'A new game-theoretic framework for maintenance strategy analysis', IEEE Transactions on Power Systems, 2003, 18, (2), pp. 698-706

[6] Kim, J. H., Park, J. B., Park, J. K., and Chun, Y. H.: 'Generating unit maintenance scheduling under competitive market environments', International Journal of Electrical Power \& Energy Systems, 2005, 27, (3), pp. 189-194 
[7] Tabari, N. M., Ranjbar, A. M., and Sadati, N.: 'Promoting the optimal maintenance schedule of generating facilities in open systems'. Proc. of 2002 IEEE Power System Technology Conference, Kumming, October 2002, pp. 641-645

[8] Wen Fushuan, Wu, F. F., and Ni Yixin: 'Generation capacity adequacy in the competitive electricity market environment', International Journal of Electrical Power \& Energy Systems, 2004, 26, (5), pp. 365-372

[9] Conejo A. J., Garcia-Bertrand R., and Diaz-Salazar M.: 'Generation maintenance scheduling in restructured power systems', IEEE Transactions on Power Systems, 2005, 20, (2), pp. 984-992

[10] State Electricity Regulatory Commission of People's Republic of China. Survey Report for Power Dispatching of Nordic and England Electricity Market. http://www.serc.gov.cn

[11] Wang, Y., and Handschin, E.: 'Unit maintenance scheduling in open systems using genetic algorithm'. Proc. of 1999 IEEE Transmission and Distribution Conference, New Orleans, LA, USA, 1999, pp. 334-339

[12] Cai Liang, and Wu Bian: ‘A regulation for congestion of generator maintenance in a deregulated system'. Proc. of 2003 IEEE Power System Technology Conference, Piscataway (NJ), June 2003, pp. 23-26

[13] Wang Jian, Wen Fushuan, Yang Rengang, Ni Yixin, and Wu, F.F.: 'Towards the development of an appropriate regulation mechanism for maintenance scheduling of generating units in electricity market environment'. Proc. of IEEE PES 2004 General Meeting, Denver, Colorado, USA, June 2004, pp. 1198-1202

[14] Gao Zhihua, and Ren Zhen: 'Competitive maintenance scheduling and settlement base on bidding in electricity market'. Proc. of 2005 IEEE Industry Applications Conference, Hong Kong, October 2005, pp. 2684-2689

[15] Lu Gang, Chung, C. Y., Wong, K. P., and Wen Fushuan: 'Unit maintenance scheduling in electricity market environment considering transmission congestion'. Proc. of the $7^{\text {th }}$ IEE International Conference on Advances in Power System Control, Operation and Management, Hong Kong, 2006 
[16] Billinton, R., and Mo Ran: 'Composite system maintenance coordination in a deregulated environment', IEEE Transactions on Power Systems, 2005, 20, (1), pp. 485-492

\section{APPENDIX}

\section{A. Congestion Rate Calculation under N-1 Examination}

The procedures of congestion rate calculation under N-1 examination are as follows:

Step 1) Analyze the historical load data of all buses and calculate the load distribution coefficient (LDC) that represents how the total system load is distributed at every bus. Forecast the maximal peak loads of all the maintenance windows, and then calculate the load of every bus in every corresponding window in terms of the LDC.

Step 2) According to the practical condition of the whole system, some key lines are determined. The set of key lines is represented by $L$.

Step 3) For a reviewed window, the injection power of each unit is sampled within the range of the relevant generation limitations, and the difference between the peak load and the total injection power is calculated. If the difference is under the maximal supply capacity outside the system, the sample is available, or else the sampling should be repeated. In more exact words, the effective sample needs to meet the following formula:

$$
\begin{aligned}
& D-\sum_{i} q_{i} \leq q_{\max }^{*} \\
& q_{i} \in\left[\underline{q}_{i}, \bar{q}_{i}\right]
\end{aligned}
$$

where $D$ is the peak load; $q_{i}$ is the random sample about the injection power of unit $i$ within the range of $\left[\underline{q_{i}}, \bar{q}_{i}\right] ;$ and $q_{\max }^{*}$ is the maximal supply capacity outside the system. Here we assume that the effective samples amount to $M$.

The above process is easy to generate useless samples because of the limitation on the supply capacity 
outside the system. In order to make the useless samples available and reduce the computation burden consequently, we make some treatment for them, i.e. randomly set $q_{i}$ at the maximization one by one until the constraint in (15) is satisfied and then make the following transformation for $q_{i}$ :

$$
\begin{aligned}
& q_{i}^{\prime}=\frac{q_{i}}{\sum_{i} q_{i}} \cdot(D-Q) \\
& \text { s.t. } \sum_{i} q_{i} \geq D-Q
\end{aligned}
$$

where $Q$ is a sample of the supply capacity outside the system within the range of $\left[q_{\max }^{*}-\sum_{i} \bar{q}_{i}, q_{\max }^{*}\right]$.

Then $q_{i}^{\prime}$ is used for the following simulation calculation.

Step 4) Carry out the DC power flow calculation in order of elements in the set $L$, and calculate the CR respectively:

$$
\begin{aligned}
& B(l) \cdot \theta=P_{G}-P_{D} \\
& F=X \theta \\
& l \in L
\end{aligned}
$$

where $P_{G}$ is the bus injection power vector; $P_{D}$ is the bus load vector; $\theta$ is the phase angle vector of bus voltage; $B(l)$ is the negative admittance matrix of rank $n$ about the outage line $l ; F$ is the line-flow vector; and $X$ is the relational matrix between the line power flow and the bus phase angle.

Count the number $M^{\prime}$ of samples for which the power flow is over in relevant windows, and the CR about the outage line $l$ is: $\gamma_{N-1}(l)=M^{\prime} / M$. 